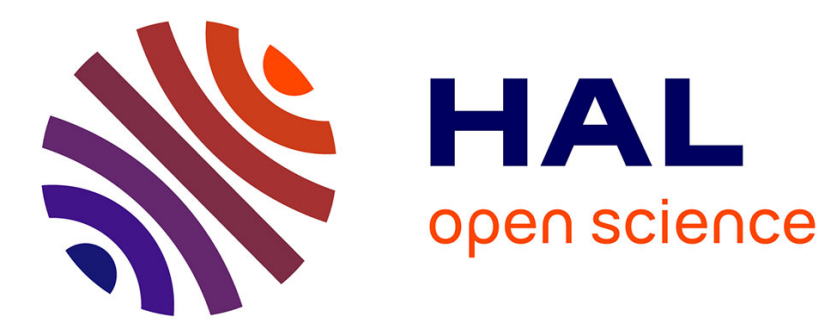

\title{
Experience of Formal Application Ontology Development to Enhance User Understanding in a Geo Business Intelligence SaaS Platform
}

Perrine Pittet, Jérôme Barthélémy

\section{- To cite this version:}

Perrine Pittet, Jérôme Barthélémy. Experience of Formal Application Ontology Development to Enhance User Understanding in a Geo Business Intelligence SaaS Platform. International Workshop Formal Ontologies Meet Industries, Aug 2015, Berlin, Germany. pp.51 - 62, 10.1007/978-3-319-215457_5. hal-01459798

\author{
HAL Id: hal-01459798 \\ https://hal.science/hal-01459798
}

Submitted on 7 Feb 2017

HAL is a multi-disciplinary open access archive for the deposit and dissemination of scientific research documents, whether they are published or not. The documents may come from teaching and research institutions in France or abroad, or from public or private research centers.
L'archive ouverte pluridisciplinaire HAL, est destinée au dépôt et à la diffusion de documents scientifiques de niveau recherche, publiés ou non, émanant des établissements d'enseignement et de recherche français ou étrangers, des laboratoires publics ou privés. 


\title{
Experience of Formal Application Ontology Development to Enhance User Understanding in a Geo Business Intelligence SaaS Platform
}

\author{
Perrine PITTET, Jérôme Barthélémy \\ Articque Software, 149 avenue Général de Gaulle, 37230 Fondettes \\ \{ppittet, jbarthelemy\}@articque.com
}

\begin{abstract}
This paper intends to share the development experience of the application ontology of the SaaS version of the decision statistical mapping and geomarketing software Cartes \& Données (C \& D). The ontology describing the C \& D application domain was conceived for automation of semantic annotation of CD7Online's users data to help users better understand their data and make better selection and representation choices. We followed the application ontology development methodology of Noy and McGuinness, which we expanded with the mapping to an upper ontology and an additional ontology evaluation step according to user specific needs.
\end{abstract}

Keywords: Cartes \& Données, application ontology, ontology development, ontology evaluation, semantic annotation, geo business intelligence

\section{Introduction}

Cartes \& Données ${ }^{1}$ (C \& D) is a decision statistical mapping and geomarketing software published by Articque ${ }^{2}$ that allows common users to obtain effective and interoperable maps, without being mapping specialists. As a business decision tool, it is a data analysis and visualization oriented application, which aims at helping people to take decisions via the maps they build upon the analysis of their geo-statistical data. Taking advantage of the new development cycle of the software-as-a-service (SaaS) version of $\mathrm{C} \& \mathrm{D}$, i.e. CD7Online, Articque decided to improve users experience to help them build meaningful maps. CD7Online is self-served, and as such needs to be self explanatory, simple, clean and highly intuitive for users. This is one of the key points to its success and it concerns both Web application ergonomics, features and data levels. To improve user experience, a recent trend, in SaaS projects, is to increase user friendliness by building user profiles and adapting application interface and services to the users preferences and context [1]. These advances have already been implemented in CD7Online, which embeds new user based features, personalized access, notably to manage user roles and rights policies, and suitable ergonomics.

\footnotetext{
${ }^{1} \mathrm{http} / / / \mathrm{www}$.articque.com/solutions/cartes-et-donnees/

${ }^{2} \mathrm{http}: / /$ www.articque.com/
} 
However, although user profiling and ergonomics allow the user to better customize the interface of the platform and facilitate their navigation, they are not enough to help them better understand their data and better assimilate the business processes of CD7Online. Actually, to compute pertinent results, CD7Online relies on the users good knowledge of their data and their ability to choose the relevant analysis and representation tools to build relevant maps. Data understanding is therefore a critical point for the success of CD7Online. Foster human understanding regarding data on the Web is the main goal of the Semantic Web [2]. To achieve this, knowledge must be extracted from data or be added thereto using a formal explicit terminology, i.e. an ontology, shared by all the users of the considered information system. Today ontologies are considered as the backbone of Semantic Web because they bridge the semantic heterogeneity gap. Therefore, to solve the data understanding issue, we decided to integrate a semantic layer to CD7Online platform to extract and represent knowledge of CD7Online user data. One of the main features of this layer is to show the users the concepts related to their data and their semantic relations, and to allow them to intuitively navigate, compare and select compatible data to build relevant maps. Domain ontologies knowledge may however not be enough complete to describe these application specificities, because that knowledge strongly depends on the CD7Online platform specific uses and processes. This implies the development of a specific application ontology.

Within this context, this paper intends to share the development experience of formal application ontology in the industrial context of the C \& D SaaS version commercial development and release. The rest of this paper is articulated in 3 sections. The second section describes the background of the CD7Online project, the reasons for the development of a formal application ontology and the criteria to take into account. The third section describes the development process within the CD7Online project and exposes the choices made at each step. The fourth section presents an analysis of the successes and drawbacks of this development experience regarding the industrial context of the project, and attempts to identify good practices for future projects.

\section{Background}

This section presents the background of the CD7Online project, such as the editor choices, the reasons of the introduction of a semantic layer and the criteria to take into account for the development of the associated ontology.

\subsection{From C \& D to CD7Online}

C \& D was first released more than 20 years ago with the main objective to make it very easy for cartographers and practitioners to build maps using their descriptive statistics data and base maps. For this purpose, a tool for building maps was implemented, in the form of a visual programming language. It enables graphically building an equivalent of the mental process used by cartographers. In the domain of 
Experience of Formal Application Ontology Development to Enhance User Understanding in a

Geo Business Intelligence SaaS Platform

mapping software, the idea was innovative in terms of ergonomics, and was already adopted in the domain of audio processing (Max/MSP$\left.{ }^{3}\right)$. The description of the tool is not the purpose of this paper. We just underline this concept has proven itself on this problem. Two years ago, in 2013, a survey was conducted with a significant panel of users of the software. For about $50 \%$ of users, the ease of use and ergonomics of the solution was the main reason for the choice of the software tool. Therefore, the ergonomics and the ease of use of the solution is not just an implementation detail for Articque, as a software publisher. It is one of the main design principles of this software tool, and even the most important one.

However, the efforts done to develop ergonomics did not resolve the problem of data identification and compatibility, which solely relies on the user's knowledge. To bridge this gap, the C \& D development team designed an online version of the $\mathrm{C} \&$ D software tool, called CD7Online, with the idea of easing the understanding and access of the users workspace. To achieve this, a semantic layer supported by an ontology-based semantic annotation was designed to extract and represent the user data knowledge, as such as the application uses and processes, via metadata. The introduction of this layer aims at encouraging CD7Online users to improve their maps by guiding them to use the right descriptive statistics data with the correct base maps, choose the right analysis tools and the best representations - for instance, by providing suggestions of base maps or data relevant to the user map project. On the social and strategic aspects, the domain terminology homogenization can help CD7Online users to better understand each other when working on the same map project. Additionally these maps' semantic annotations, which are used to identify the semantic relations between maps, allow reinforcing the user community by suggesting working groups to users having published closely related maps.

\subsection{Semantic Annotation in CD7Online and Application Ontology}

Automatized semantic annotation of data is the process of automatically associating relevant metadata to data, so that each data is described by a set of semantic annotations. According to [3], a practical semantic annotation is impossible without some particular knowledge modelling commitments. i.e. an ontology. They add that a system for semantic annotation should be complemented with extensive instance knowledge. In our case, the knowledge model must describe all the metadata used to annotate the $\mathrm{C} \& \mathrm{D}$ user data and the extension must contain all the corresponding instances. For example, GeographicalLevel is a metadata describing the geographical division level used by a map. Its definition, including semantic relations and logical rules, is stated by the knowledge model. Its instances, such as regional level, department level or city level, are used to annotate user data files, w.r.t. this definition. Also, in order to help users understand their data annotations, corresponding terms have to describe the application domain, uses and processes in an explicit and consensual way, so that they can be shared and used by all the users of the application. The definition of a formal application ontology as such knowledge

\footnotetext{
${ }^{3}$ http://en.wikipedia.org/wiki/Max_(software)
} 
model definitely suits this requirement. According to [4] an application ontology is an ontology engineered for a specific use or application focus and whose scope is specified through testable use cases.

For the purpose of this article, we rely on the ontology definition of [5]. Therefore, we define the application ontology as a formal explicit description of concepts of the C \& D user data domain, properties of each concept describing various features and attributes of the concepts, and restrictions on properties. The ontology together with the set of individual instances constitutes the knowledge base designed for the automatized semantic annotation process. For example, a concept of DataIndicator represents all the data indicators, which can qualify descriptive statistics data of a user data storage. We can specify specific indicators as instances of this concept. For example, «rate» is an instance of the concept DataIndicator. A concept can have subconcepts representing concepts that are more specific than this concept. For example we can specialize the concept of UserData, into the subconcepts StatisticalData, GeographicalData and Maps. Properties describe properties of concepts and instances. For example, we can describe instances of StatisticalData as composed of DataColumn instances, which have a title, and are related to a DataIndicator instance. Here we have three properties: the column property describing a StatisticalData instance with an instance of DataColumn, the title and the dataIndicator properties describing a DataColumn instance with the respective values: a literal value and an instance of DataIndicator. For example, we can specify the instance ventes2014 of StatisticalData as a data file composed of three columns, which have for titles «City code», « Nb Inhabitants » and « Sales », and respectively have for related data indicators the instances city_code, population and turnover. We chose to specify the ontology in OWL DL language to keep the maximum expressiveness possible while retaining computational completeness, decidability and the availability of practical reasoning algorithms.

\subsection{Ontology Development Methodology}

Many methodologies have been proposed in the literature to help ontology developers as described in [6], [7], and [8]. Globally, besides the identification of some key development steps, ontology development appears to be a creative, iterative and collaborative process, which implies the participation of different people with different points of view on the domain to describe. Therefore the potential applications of the ontology and the designers' understanding and view of the domain undoubtedly affects ontology design choices. According to [9], in practical terms, developing an ontology includes: defining concepts in the ontology, arranging the concepts in a taxonomic hierarchy, defining properties and describing allowed values for these properties, and filling in the values for properties for instances. Then a knowledge base, defining individual instances of these classes filling in specific properties value information and additional property restrictions, can be created. Ontology correction and evolution is often required to adjust the terminological description to undetected or new requirements especially in the case of application ontologies. Also the choice of a specific ontology development methodology depends 
Experience of Formal Application Ontology Development to Enhance User Understanding in a

Geo Business Intelligence SaaS Platform

on the possible uses of the ontology (semantic heterogeneity resolution, domains fusion, inference, etc.), the level of detail (general, domain or application level) that is necessary for the application, personal preferences, and sometimes requirements for compatibility with other models (ontology integration).

\section{Ontology Development Process}

For CD7Online semantic annotation purpose, we chose to follow a simple knowledge engineering methodology proposed by Noy and McGuinness [9], dedicated to the development of application ontologies. The 7 steps of this methodology are described and illustrated through the presentation of the CD7Online application ontology development process in the following parts.

\subsection{Determination of the domain and scope of the ontology}

This step aims at determining the domain and scope of the ontology by answering the following questions: (1) what is the domain the ontology will cover? (2) For what are we going to use the ontology? (3) For what types of questions the information's in the ontology should provide answers? (4) Who will use and maintain the ontology? For this first step, a pre-analysis of the domain was realized in partnership with the C \& D domain experts: C \& D development, product support, training, business, geomarketing, and cartography teams. This pre-analysis of the domain consisted in interviews with members of each team about its expert subdomain to delimit the domain the ontology will describe. We managed to gather many user feedbacks, which helped us to precise the applications of the ontology and therefore guide the ontology conception choices. We then answered the questions above: (1) the ontology will describe the CD7Online user data including descriptive statistics data files (tables of data), geographical data files (base maps) and maps projects (organization charts), but also the platform related business processes and uses; (2) this ontology will be used as a model for the automatized semantic annotation of the user data; (3) it should provide answers to the following questions: What do users data represent (in terms of the domain concepts)? What do they contain? How are they linked? What is their type? How are they used? What is their scope? How can these data be used?; (4) this ontology will be used by the CD7 online version of the C \& D software tool for the automatized annotation of user data and recommendation of semantically related data, projects, groups. CD7Online users will only interact with the semantic annotations and recommendations. The ontology will be maintained by the application developers and supported by the application domain experts.

\subsection{Consideration of the reuse of existing ontologies}

We extended this second step of considering reuse of existing ontologies to searching out various representative dictionaries related to the domain of interest as recommended by [10]. We identified two means of finding those relevant resources. 
First we searched domain ontologies, embedding dictionaries with strong formal semantics, which have been developed with the goal of being reused by users of the domain they describe for their application needs. The domain to be covered by the $\mathrm{C}$ $\& \mathrm{D}$ application ontology includes the definitions and relations between descriptive statistics data, geographical data (base maps), map projects and their usages within C \& D. To cover this domain, we selected: indicators from the general statistics ontology $\mathrm{STATO}^{4}$, quantities, units, and dimensions from $\mathrm{QUDT}^{5}$ ontology, geographical properties from GeoNames ${ }^{6}$, and concepts of map representation and symbolization process from cartographic ontologies as presented in [11]. Second, we searched the CD7Online development project environment resources. With the help of the C \& D development and cartography teams, we actually found knowledge models of public descriptive statistics data indicators and geographical data (base maps). Descriptive statistics data indicators are classified and available in the MapAndData ${ }^{7}$ frontend database. Their classification takes into account the geographic spaces, the geographic levels, the data projection, and the related themes. The MapAndData database administrators have also built a dictionary of the corresponding data indicators for each theme and manually annotated them with the right data type, units and geographical levels. This dictionary of metadata on the public data contains the basic vocabulary and describes the basic relationships between metadata. User personal descriptive statistics data are expected to meet the same data indicators and themes if used in conjunction with these public data. Analysis of maps files created by C \& D users, allowed extracting their structure, which is specific to $\mathrm{C} \& \mathrm{D}$. A map contain an organization chart, which is a graph representing the modules used to bind data to base maps, process them and render them within a $2 \mathrm{D}$ visualization. Also, searching the $\mathrm{C} \& \mathrm{D}$ specifications, we found that all maps components and rules are already defined. Once relevant domain ontologies and resource models are identified, we manually sift through those dictionaries in order to extrapolate all of the relevant terms for that domain. We obtained a rough-draft, resource lexicon like recommended by Guarino [12], which is a blend of other disparate sources of information.

\subsection{Lexicon of important terms in the ontology}

The third step consists in building a rough lexicon of important terms related to the domain. We chose to identify these terms by defining the application domain data and processes in natural language. In the following description, these terms are written in italic. As stated before, the application ontology focuses on the description of C \& D user data. Three types of user data are considered for semantic annotation: descriptive statistics data, geographical data and maps. The definition of the term user data required delving into other subordinate definitions such as user, data, statistical data,

\footnotetext{
${ }^{4} \mathrm{http}: / /$ stato-ontology.org/

${ }^{5} \mathrm{http}: / / \mathrm{www}$.qudt.org/

${ }^{6} \mathrm{http}: / /$ www.geonames.org/ontology/documentation.html

${ }^{7} \mathrm{http}: / /$ www.mapanddata.com/
} 
Experience of Formal Application Ontology Development to Enhance User Understanding in a Geo Business Intelligence SaaS Platform

geographical data etc. Understanding the term user data therefore depends on understanding these related terms. Statistical data also called data tables are both public and user private statistics, which are owned by the users and stored on their data repository. These tables are composed of columns of data with at least one id column. Each column has a title and corresponding type and data indicator. A column can have a date but also a unit if its type is numeric. The id column name is a geographical code data indicator, which indicates the geographical level considered by the whole table data. Data results and column titles are obviously recognizable in tables whereas the extraction of other information requires a natural language processing extraction approach to be applied. We have chosen a structural data semantic annotation methodology, like proposed by [13] using semantic similarity rules, to decompose each table cell content into French lemmas, and compare them with weighted words, composing weighted terms used in the domain, and associated to data indicators. Geographical data also called base maps are also owned by the users and are used to represent descriptive statistics data results on a geographical map. They are specified in a proprietary format, which can only be processed by $\mathrm{C} \&$ D. Therefore their corresponding date, geographical space and level cannot be extracted from their content, but appears in their filename. Maps also called $C \& D$ projects are the result maps of the representation of statistical computations on base maps. Maps contain an organization chart composed of relations between modules. These relations enable binding statistical and geographical data with calculation, statistical and geographical operators and representations. A module takes a statistical or geographical data as input and display calculated descriptive statistics data, representations or visualizations in output. Modules are classified in C \& D according to their use purpose (data sources, geographical, statistical and hybrid data operators, representations and visualizations). According to Little and Engineer [10], once the rough-draft lexicon was compiled, each term in that lexicon was examined in order to uncover merged or composite terms, whose definitions contain secondary terms which themselves require definition.

\subsection{Definition of the concepts and concept hierarchy}

Once the lexicon of terms is considered complete and consistent, we followed the recommendations of Little and Engineer [10] and selected a set of inter-related upperlevel categories from the foundational ontology DOLCE [14]. This part of the ontology development process was not only a mapping exercise to this upper ontology. It provided the C \& D ontology an abstract, philosophical basis, which is crucial for its design, implementation and reuse and under which every specific category within the ontology falls. Although the C \& D ontology is not meant to be reused for the moment, we do not foreclose this eventuality in the future. We also believe this metaphysical foundation may ease the potential integration of other formal ontologies. We chose the DOLCE foundational ontology [14] (cf. Fig.1) because it is a solid starting point for building new formal ontologies. The mapping consisted in three steps. We first identified the most general concepts among our list of terms: User and Component. Second, we defined them precisely: A User is a 
person who has a user account on the CD7Online platform; a Component is a component of any type, which can be related either to user data (DataComponent) either to the CD7Online specific application processes (CDComponent). Third we determined the categories under which they fall. User and Component are Endurant according to the DOLCE upper level categories because they are independent essential wholes. They are Non-physical object as they have no direct spatial qualities (no weight, etc.). They are both Social object as they are entities from the social world. Social object includes both agentive and non-agentive socially constructed objects. User can be categorized as an Agentive social-object and more precisely as a Social Agent as a user is assumed to have intensions, beliefs and desires. Inversely, Component is a Non-agentive social object and more precisely as an Information object it can be realized by some entity, ordered by some system for information encoding. It can express a description, can be about any entity, and can be interpreted by an agent.

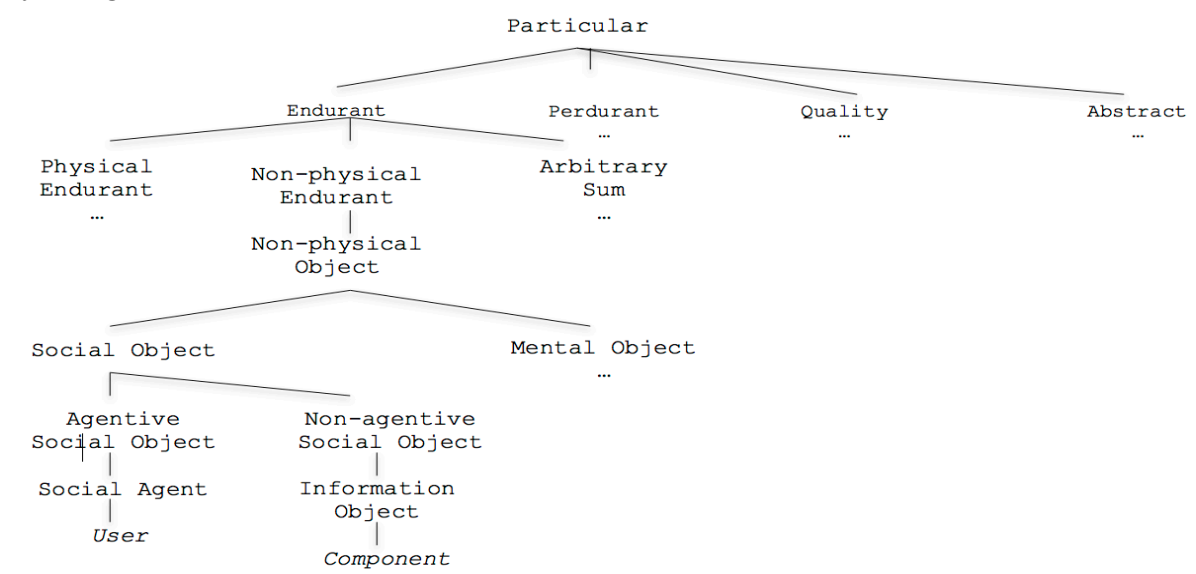

Fig. 1. Upper level categories of CD7Online ontology reusing DOLCE

Once the upper-level taxonomy in place, the domain and application-specific categories were derived from the ontology's lexicon. However, many of the domainspecific categories needed for the ontology were not exhaustedly defined by the set of terms within the lexicon. Many terms' definitions contain subsequent terms within them, which also needed to be categorized within the ontology. For instance, Statistical data is defined as user data, which have at least one id column and one data column. Below is the corresponding description logic definition [15]:

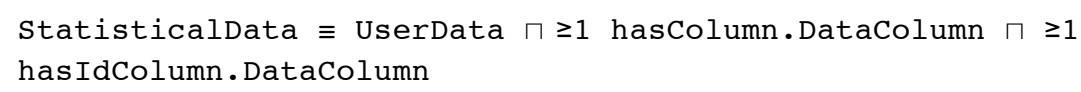

This definition implies to define the UserData and DataColumn concepts. User data are data files owned by a user with a filename expressed as a string, and they can have a geographical level and/or a geographical space. Here is the corresponding definition: 
Experience of Formal Application Ontology Development to Enhance User Understanding in a Geo Business Intelligence SaaS Platform

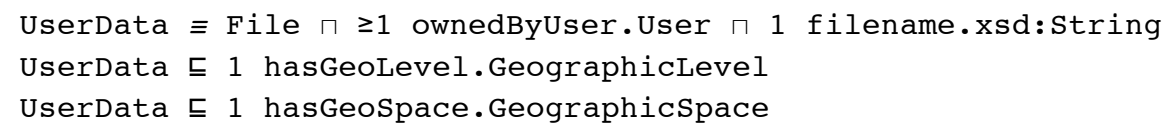

These definitions lead us to define the terms File, GeographicLevel and GeographicSpace, etc. All the terms' definitions describe a hierarchy of terms extending the upper-level categories hierarchy (cf. Fig. 1). For instance the following definition of Metadata, which are information of user data obviously specified or semantically extracted from UserData.

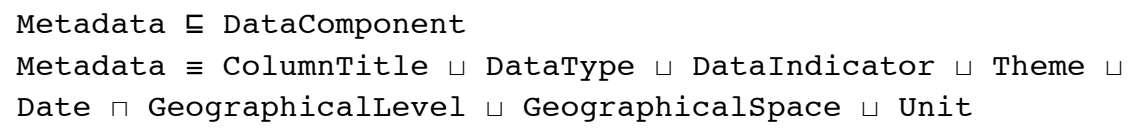

This definition implies an underlying concept hierarchy, represented in Fig. 2 below.

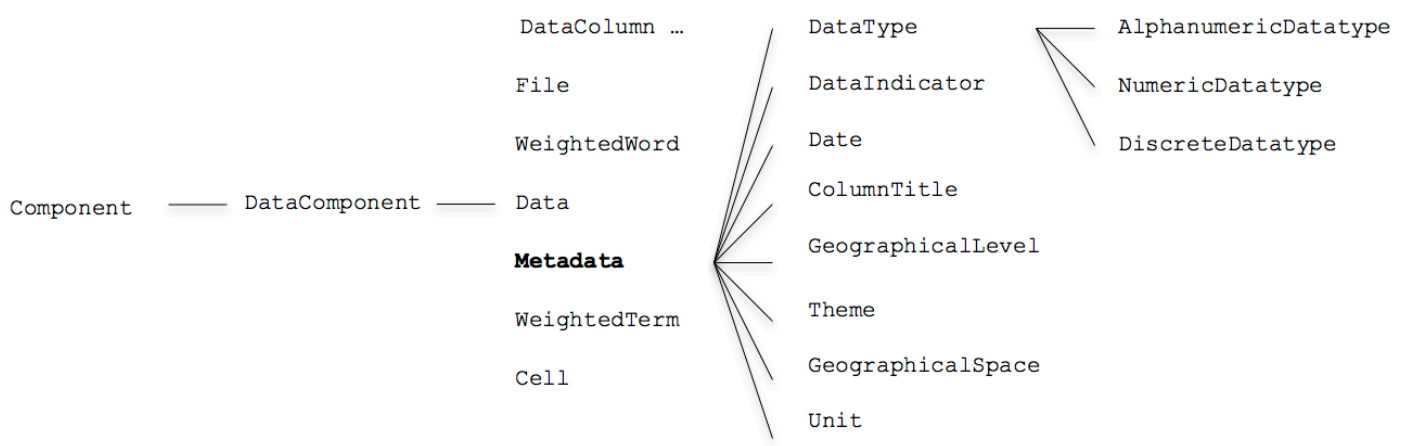

Fig. 2. Underlying concept hierarchy of Metadata

\subsection{Properties Definitions and facets}

Once the whole concept hierarchy was established, the properties domain and ranges, and their facets could be defined. For instance the ownedByUser property is an object property, which describes a relation of ownership between a file (domain) and a user (range). According to the notations used in [16], the domain and range of the ownedByUser property are defined below:

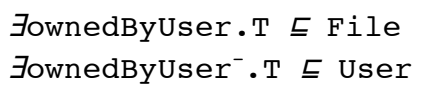

Among the facets to define, the property cardinalities restrict the number of range or domain instances a property can respectively have for a same instance in the domain or a same instance in the range. For example, the ownedByUser property was restricted by:

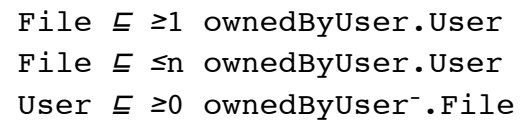


User $\underline{\subseteq} \leq \mathrm{n}$ ownedByUser-File

\subsection{Creation of Instances}

After defining the terminology, the remaining step was to create the required individual instances of concepts in the hierarchy to setup the knowledge base for our needs. Instances of the concepts WeightedWord, WeightedTerm, DataIndicator, Datatype, Themes, Units, GeographicalLevel, GeographicalSpace were required for the automatized semantic annotation process. For example, to identify and annotate a data column with a data indicator and a theme, the process evaluates the similarity of data cells content with weighted words, which are composed of weighted terms associated to data indicators, themselves classified in themes. Instances of all these concepts were stated according to the MapAndData database compilation. Defining an individual instance of a concept requires (1) choosing a concept, (2) creating an individual instance of that concept, and (3) filling in the properties values. Below is an example of instances creation to setup the rate indicator for semantic annotation:

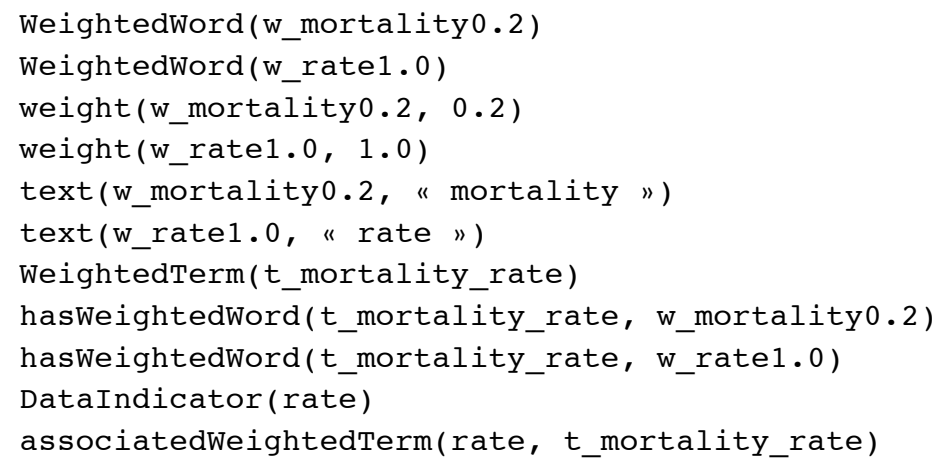

In addition to the ontology development steps listed by [9], we added another step that we believed important for our purposes: ontology evaluation.

\subsection{Ontology Evaluation}

As stated in [10], ontology evaluation represents the most difficult step because it amounts to an elaborate feedback mechanism, nearly identical to the form of feedback found within life-cycle models in other areas of systems engineering. Subhashini and Akilandeswari [7] recommend evaluating the constructed ontology by taking some evaluation criteria. These criteria can be categorized into two types, namely generic criteria and specific criteria. The generic criteria deal with factors like clarity, consistency, and reusability. The specific criteria check the generated ontology against the purpose and user requirements. Concerning clarity, it has been assessed by the C \& D teams members. Consistency was evaluated by the Pellet reasoner. Reusability was not measured as, for the moment, the ontology is only designed for $\mathrm{C}$ $\& \mathrm{D}$ application semantic annotation purposes. To assess the ontology against specific criteria, we needed to deploy the ontology on an environment similar to the 
Experience of Formal Application Ontology Development to Enhance User Understanding in a

Geo Business Intelligence SaaS Platform

production one in order to test it on real data. As we said, ontology development is an incremental process, during which the ontology is refined until its condition allows it to achieve the purpose for which it was developed. Refining the ontology actually tended to increase the number of axioms and therefore the size of the ontology. Therefore, we had to load the ontology in a triplestore instead of loading the ontology in memory each time it has to be queried. We chose the Fuseki triplestore because of its compatibility and ease of use with both Java and PHP technologies, which are implemented in CD7Online. Within this environment several real case sets of data, base maps and maps have been tested after each semantic annotation process by assessing the annotations correctness and consistency such as data type, statistical indicator, unit, year, geographic level and geographic area. For instance the percentage of good annotation of data with statistical indicators obtained at the first evaluation round on a set of 571 data columns was $35.2 \% .38 .4 \%$ data columns were not annotated because no indicator was identified or the system could not choose between several ones. $17.7 \%$ annotations were non-optimized and $8.8 \%$ wrong, because certain indicators were not modelled or too specific. The ontology was revised to obtain better results and we have fixed an objective of $80 \%$ good results. Also, regarding the business inherent necessity to obtain quick results within short deadlines, the ontology revision process had to be stopped and restarted several times to keep pace with the development runs. The last revision permitted to reach $82.5 \%$ of good annotations. Tests are not finished yet; the application ontology is integrated into a beta version of the CD7Online application, which is still tested by a limited set of users.

\section{Conclusions and future works}

In this paper, we first presented the CD7Online project and explained why it needs a semantic layer to help its users better understand their data and the platform features. We also justified the development of an application ontology to support the automated semantic annotation of the CD7Online user data. Second we presented the different phases of the application ontology development following the application ontology development methodology of Noy and McGuiness [9]. This experience exposed several issues concerning the development of a formal ontology in an industrial project, namely the lack of time. Ontology development is a collaborative labour-intensive manual task, which requires a meticulous analysis phase, comprising the study of various representative dictionaries related to the domain of interest, and a conceptual modelling phase with many refinement stages to increase the ontology soundness and completeness. We try to meet these requirements, in the context of the CD7Online product release, through the allocation of a full time resource on this task for several months. To improve and facilitate the evaluation of this ontology, we would recommend a specific benchmark embedding a corpus of diversified descriptive statistics data, base maps and maps dedicated to C \& D software tool, and a list of evaluation criteria such as annotation correctness and consistency average, precision, scalability. Today CD7Online provides users with an interactive 
visualization tool designed for intuitively navigate through data via metadata, built on the top of the semantic layer. Beyond this first application, we envision in the future the development of a recommendation tool, aiming at sharing good practices, data or base maps, or working groups, the main idea being to make easier and more efficient the use of the CD7Online software tool.

\section{References}

1. Tsai, W. T., Shao, Q., \& Li, W. (2010, December). Oic: Ontology-based intelligent customization framework for SaaS. In Service-Oriented Computing and Applications (SOCA), 2010 IEEE International Conference on (pp. 1-8). IEEE.

2. Berners-Lee, T. (2000). Semantic Web Stack.

3. Kiryakov, A., Popov, B., Terziev, I., Manov, D., \& Ognyanoff, D. (2004). Semantic annotation, indexing, and retrieval. Web Semantics: Science, Services and Agents on the World Wide Web, 2(1), 49-79.

4. Malone, J., \& Parkinson, H. (2010). Reference and application ontologies. Ontogenesis.

5. Gruber, T. R. (1993). A translation approach to portable ontology specifications. Knowledge acquisition, 5(2), 199-220.

6. Corcho, O., Fernández-López, M., \& Gómez-Pérez, A. (2003). Methodologies, tools and languages for building ontologies. Where is their meeting point?. Data \& knowledge engineering, 46(1), 41-64.

7. Subhashini, R., \& Akilandeswari, J. (2011). A survey on ontology construction methodologies. International Journal of Enterprise Computing and Business Systems, 1(1), 60-72.

8. Suarez-Figueroa, M. C., Gomez-Perez, A., \& Fernandez-Lopez, M. (2012). The NeOn methodology for ontology engineering. In Ontology engineering in a networked world (pp. 9-34). Springer Berlin Heidelberg.

9. Noy, N. F., \& McGuinness, D. L. (2001). Ontology development 101: A guide to creating your first ontology.

10. Little, E., \& Engineer, O. (2003, September). A Proposed Methodology for the Development of Application-Based Formal Ontologies. In KI Workshop on Reference Ontologies and Application Ontologies.

11. Iosifescu-Enescu, I., \& Hurni, L. (2007, August). Towards cartographic ontologies or" how computers learn cartography. In Proceedings 23rd International Cartographic Conference (pp. 4-10).

12. Guarino, N. (1995). Formal ontology, conceptual analysis and knowledge representation. International journal of human-computer studies, 43(5), 625-640.

13. Hignette, G. (2007). Annotation sémantique floue de tableaux guidée par une ontologie (Doctoral dissertation, AgroParisTech).

14. Masolo, C., Borgo, S., Gangemi, A., Guarino, N., Oltramari, A., \& Schneider, L. (2003). WonderWeb Deliverable D17. The WonderWeb Library of Foundational Ontologies Preliminary Report. ISTC-CNR, Padova. URL http://wonderweb. semanticweb. org/deliverables/documents D, 17.

15. Baader, F., \& Nutt, W. (2003, January). Basic description logics. In Description logic handbook (pp. 43-95).

16. Zhang, R., Artale, A., Giunchiglia, F., \& Crispo, B. (2009). Using description logics in relation based access control. 